Musées, Patrimoine et Culture scientifiques et techniques

$167 \mid 2016$

septembre-octobre 2016

\title{
Un échange de pratiques entre institutions patrimoniales
}

\author{
Gaëlle Lesaffre
}

\section{OpenEdition \\ Journals}

Édition électronique

URL : http://journals.openedition.org/ocim/1700

DOI : $10.4000 /$ ocim. 1700

ISSN : 2108-646X

\section{Éditeur}

OCIM

Édition imprimée

Date de publication : 1 septembre 2016

Pagination : $30-39$

ISSN : 0994-1908

\section{Référence électronique}

Gaëlle Lesaffre, «Un échange de pratiques entre institutions patrimoniales », La Lettre de I'OCIM [En ligne], 167 | 2016, mis en ligne le 01 septembre 2017, consulté le 30 avril 2019. URL : http:// journals.openedition.org/ocim/1700 ; DOI : 10.4000/ocim.1700

Ce document a été généré automatiquement le 30 avril 2019.

Tous droits réservés 


\section{Un échange de pratiques entre institutions patrimoniales}

\section{Gaëlle Lesaffre}

À Montréal, présentation des "Brigades mobiles" par l'équipe du Rize

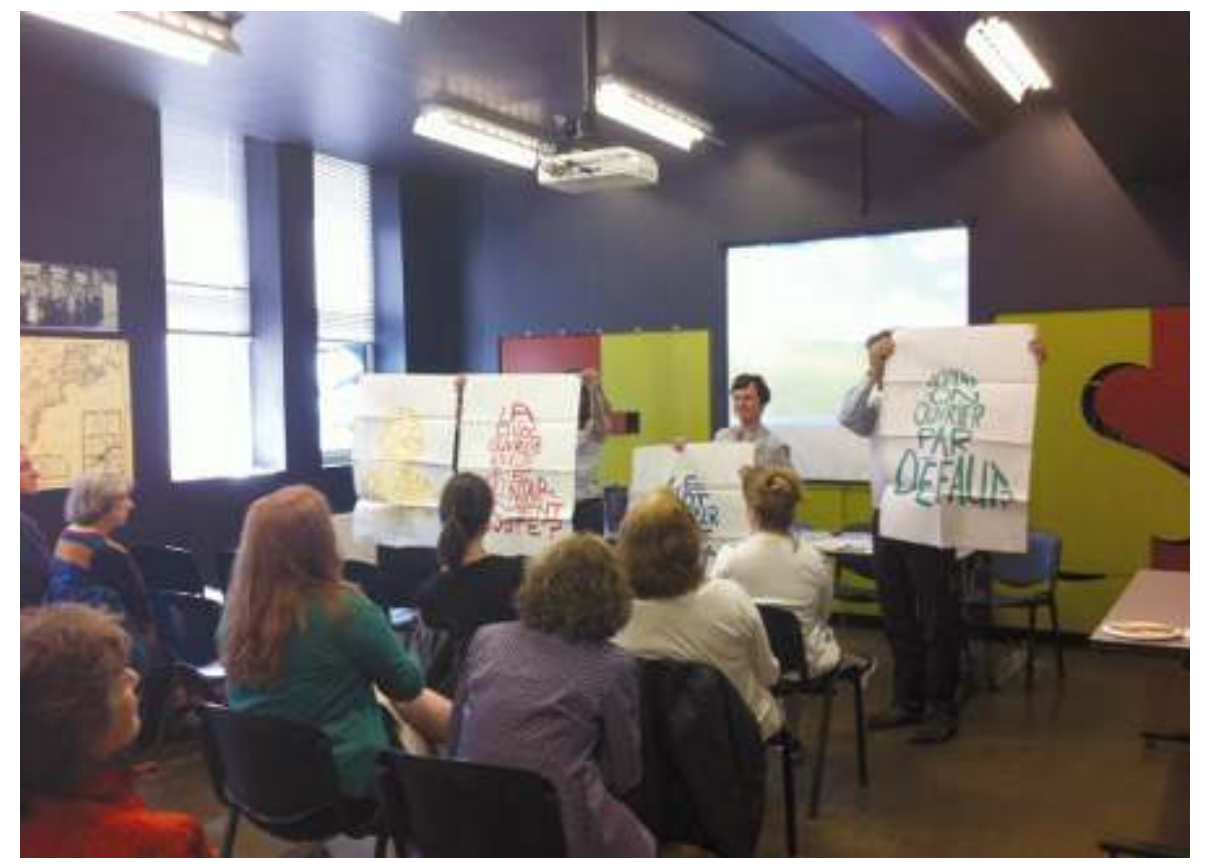

(c) G. Lessaffre

1 Comment créer les conditions de la rencontre et de l'échange entre des équipes professionnelles, qui plus est lorsqu'elles sont situées de chaque côté de l'Atlantique, l'une à Villeurbanne en France, l'autre à Montréal au Québec? Le défi du partage de pratiques professionnelles a été relevé par l'équipe du Rize, lieu culturel municipal dédié à la construction d'un récit collectif sur le territoire villeurbannais, notamment à travers les mémoires ouvrières et de l'immigration, qui compte aussi une médiathèque et les 
Archives municipales, et celle du Centre d'Histoire de Montréal (CHM), centre d'interprétation de l'histoire de Montréal rattaché à la municipalité.

\section{Un échange construit sur plusieurs années et soutenu par l'Accord France-Canada}

2 Le projet d'échange entre les équipes du Rize de Villeurbanne et du Centre d'Histoire de Montréal a abouti en 2015, après plusieurs années de maturation et de construction.

3 En quoi ce projet pouvait être effectivement un échange, et pas seulement en porter le nom? Et que pouvaient en attendre les partenaires? De manière générale, l'échange social se caractérise par deux dimensions principales. D'une part l'engagement des partenaires dans l'échange est motivé par l'espoir qu'il produise quelque chose. D'autre part, ce qui est échangé n'est pas connu à l'avance, inscrivant l'échange dans le temps et dans la confiance. A priori, l'échange entre le Rize et le Centre d'Histoire de Montréal était bien fondé sur un engagement des partenaires, mais à travers les deux directeurs, motivés par l'espérance que cet échange apporte "quelque chose" à leurs deux institutions. L'adhésion des équipes au projet, l'instauration d'une relation de confiance et ce qui allait être produit par l'échange constituaient autant d'inconnus dans la réalité et finalement dans la réussite de l'échange.

4 En 2012, les directeurs des deux institutions se rencontraient au sein d'un groupement d'intérêt scientifique soucieux de valoriser les démarches patrimoniales interculturelles : le Gis Ipapic. Aujourd'hui dissout, il réunissait des responsables d'institutions culturelles dans le champ des musées et du patrimoine, des archives et des bibliothèques; des chercheurs et des associations impliquées dans les questions patrimoniales ${ }^{1}$ et dans une démarche favorisant l'échange entre les représentants des institutions culturelles, les chercheurs et les représentants des associations. L'échange, l'interculturalité des pratiques, le souci de penser différemment le rapport au patrimoine étaient donc inscrits dans la rencontre.

5 Dès 2012, la proposition du directeur du Rize de collaborer dans le cadre de l'Accord France-Canada pour "la coopération et les échanges dans le domaine des musées"2 est envisagée par les deux directeurs. Le directeur du CHM se rend pour la première fois au Rize en septembre 2013 pour prendre connaissance des lieux et du projet. Par la suite, de nombreux échanges, à distance, permettent de dresser les contours du projet et, en août 2014, le projet de partage de pratiques "L'histoire, la mémoire et le patrimoine au cœur de l'animation urbaine" est retenu par le comité de l'Accord France-Canada, donnant un cadre formel et un soutien financier au projet.

\section{Une grande réciprocité des formes de l'échange au Québec et en France}

6 La forme de l'échange prévue par le projet impliquait le déplacement de trois membres de l'équipe d'une institution pendant une semaine dans l'institution hôte et vice versa. Les deux volets de l'échange devaient être suivis par une chercheuse en sciences humaines français. Celle-ci a été mise en position d'observation non participante, donc mobilisée pour ses compétences en termes d'observation et d'analyse de l'interaction, et non pour 
sa participation sur le fond de l'échange, se détachant ainsi de la démarche du Gis Ipapic, à l'origine de la rencontre 3 . Cependant, elle a constitué l'élément clé qui a permis à ce projet répondant à des objectifs pragmatiques, de rejoindre, grâce à une démarche plus classique d'observation et de recherche, un objectif fondamental du Gis: "mieux (se) comprendre pour dessiner des pistes d'action tant en matière de projet d'établissement que de politiques culturelles".

Au Rize, le Centre d'histoire de Montréal présente les "cliniques de la mémoire".

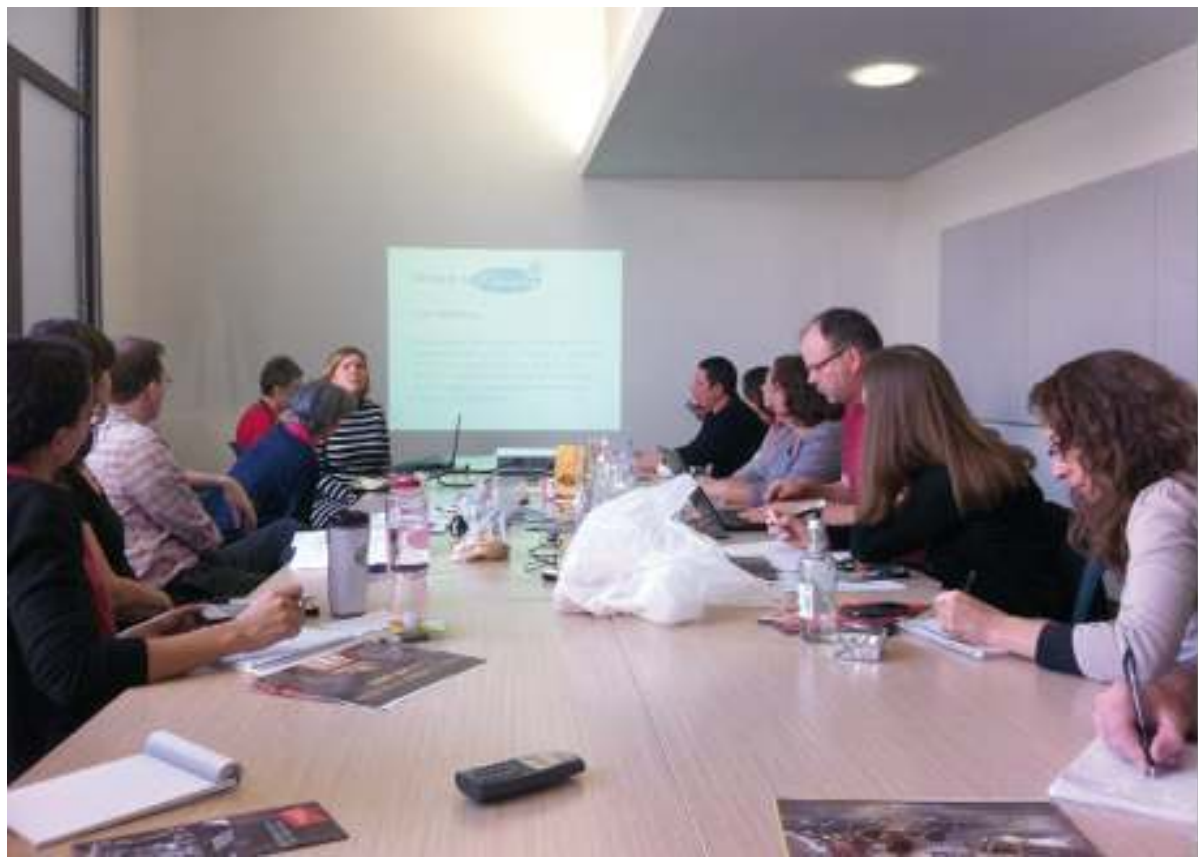

(c) G.Lesaffre

7 L'équipe du Rize s'est déplacée la première à Montréal au printemps 2015. Elle était formée de la responsable des expositions, de la responsable du développement des publics et des projets partagés et de la responsable des actions de médiation, venue à la place du directeur en raison de son départ de l'institution pour la direction des musées Gadagne de Lyon. L'équipe réunie devait donc poursuivre le projet sans son premier instigateur.

En automne 2015, l'équipe du CHM venue à Villeurbanne a réuni une muséologue, une agente de programmes éducatifs et un guide animateur, également chargé du centre de documentation, venu lui aussi remplacer le directeur du CHM retenu par le projet d'ouverture d'un nouveau bâtiment et donc d'un nouveau programme pour le CHM. Les changements de participants, conséquences de la construction du projet d'échange sur le temps long, n'ont donc finalement pas affecté l'équilibre qui avait été construit initialement. L'équilibre a même été reconstruit en mobilisant, à quelques différences près ${ }^{4}$, les alter ego des deux équipes.

9 L'équipe québécoise a donc conçu la première un programme de cinq jours. Elle a volontiers accueilli les attentes formulées par les Français de rencontrer, par exemple, l'écomusée du Fier Monde, et laissé des plages libres dans l'agenda jusqu'à leur arrivée à Montréal. Le programme prévoyait des temps d'observation des activités menées par l'institution auprès $\mathrm{du}$ public, de présentation de chaque structure accueillie à l'institution accueillante et vice versa, de visites de l'institution et d'autres institutions sur 
le territoire, un temps de présentation de projets d'autres institutions partenaires ouvert aux professionnels, un temps d'atelier et des temps conviviaux. Le programme de la semaine villeurbannaise a comporté les mêmes types d'activités, permettant d'entretenir, là encore, la réciprocité de l'échange des deux côtés de l'Atlantique.

\section{Une appropriation différente du projet par les équipes en fonction des circonstances}

10 L'appropriation du projet par les professionnels impliqués dans l'échange a évolué au gré des circonstances, et notamment de l'annulation de la venue des directeurs, de l'ordre dans lequel les équipes ont été accueillies ou accueillantes et de la difficulté pour les professionnels accueillants de se rendre disponibles tout au long du programme.

11 Initialement, le projet a été conçu par les deux directeurs. L'annulation de la venue du directeur du Rize au Québec, en raison de son départ de l'institution, a provoqué le report des enjeux de la rencontre entièrement sur les autres membres de l'équipe, même si un dialogue avait permis auparavant de les transmettre et de les partager. Les membres de l'équipe se sont alors emparés de ces enjeux au point de ressentir une moindre implication de la part de l'équipe d'accueil, et de regretter, à l'issue de la première semaine, le peu de temps informels partagés susceptibles de favoriser la rencontre individuelle en dehors du cadre défini, manque également ressenti, a posteriori, par l'équipe du CHM lors de cette première semaine.

12 L'appropriation a, en effet, été plus longue pour l'équipe du CHM qui a accueilli le Rize en premier. Cela est dû en partie au fait que les équipes n'ont pas été intégrées de la même manière au projet. Au CHM, celui-ci est resté essentiellement porté par le directeur jusqu'à ce que sa participation à l'échange à Villeurbanne soit annulée à son tour. S'ajoute à cela le fait que l'équipe qui accueille en premier, comme cela a été le cas du CHM, ne connaît pas l'autre institution; tandis que les trois membres de l'équipe villeurbannaise ayant participé au voyage connaissaient les lieux, le contexte et l'équipe du CHM au moment de la recevoir. Enfin, il est parfois difficile pour les participants accueillants restés dans leurs institutions, donc dans la gestion et les activités quotidiennes, de se rendre disponibles. Les activités auprès des publics doivent être réalisées, une communication prévue dans un colloque ne peut être repoussée. Cela a pu être observé à Montréal comme à Villeurbanne.

\section{Le déroulement de l'échange et sa réception}

13 Les programmes proposés à Montréal et à Villeurbanne étaient très proches en termes de rythme et d'activités, ce qui permet de penser que la pertinence de la forme du programme initialement prévu, tant dans les respirations qu'elle proposait que dans les moments d'échanges, de travail, de visites, a été reconnue.

De chaque côté, la semaine a débuté au contact direct de l'action de l'institution hôte et de ses publics : par la restitution aux enfants des projets auxquels ils ont contribué dans le cadre du programme "Vous faites partie de l'histoire" au CHM ; par la visite sensorielle du quartier des Buers menée par les enfants de l'école Jean Moulin à Villeurbanne. Cette plongée dans le vif du sujet a marqué les participants et leur a permis à la fois de 
percevoir rapidement le sens de l'action menée par l'institution partenaire et d'en constater les affinités avec leur propre pratique.

Les moments de présentation des institutions accueillantes et accueillies intervenus tôt dans la semaine, et ouverts à l'ensemble des personnels des institutions accueillantes, ont permis à chacun de prendre connaissance de l'autre, c'est-à-dire : de son environnement géographique immédiat (centre-ville historique et touristique pour le CHM, plus périphérique pour le Rize), politique et institutionnel (service et équipement municipaux, multiplication des musées d'histoire de Montréal à Montréal), de ses moyens, notamment humains (similaires sauf pour le poste de "développement des publics", et en dehors du personnel des archives et de la médiathèque du Rize), de ses missions et de ses projets (conservation de collections matérielles et immatérielles, expositions permanentes et temporaires mais de longue durée et activités éducatives pour le CHM, recueil et valorisation de la mémoire de la ville, ouvrière et de l'immigration à travers des actions pédagogiques ouvertes au grand public et des expositions), et ont constitué un moment privilégié pour se comparer et prendre la mesure des points communs et des différences. Les visites au sein de l'institution accueillante ont permis de prolonger ces échanges en rendant compte concrètement de l'organisation, des activités et des équipements disponibles, comme la salle de spectacle-auditorium et la réunion avec la médiathèque et les Archives municipales pour le Rize, là où le CHM dispose d'une exposition permanente ; des bureaux dans le bâtiment pour le Rize, dans un bâtiment séparé pour le CHM...

Les visites d'autres institutions, et les intervenants invités à présenter des projets de leur institution - choisis parmi les partenaires de l'institution accueillante par affinités avec le sujet de l'échange et les pratiques de l'institution accueillie - ont permis d'ouvrir aux équipes accueillies les portes de partenaires sur le territoire de chacun ${ }^{5}$ et d'y trouver parfois les éléments "manquant" chez les acteurs de l'échange. Ainsi, le musée Gadagne de Lyon, musée d'histoire de la ville implanté dans le centre-ville historique et touristique de Lyon et proposant une exposition permanente, a apporté ces éléments de comparaison au CHM ; tandis que l'écomusée du Fier monde a apporté au Rize une approche tournée vers les "citoyens" hors des circuits touristiques et centrée sur l'histoire et le patrimoine ouvrier et industriel. 
Premier contact des Montréalaises avec le Rize de Villeurbanne : la visite du quartier des Buers par des élèves de l'école Jean Moulin de Villeurbanne

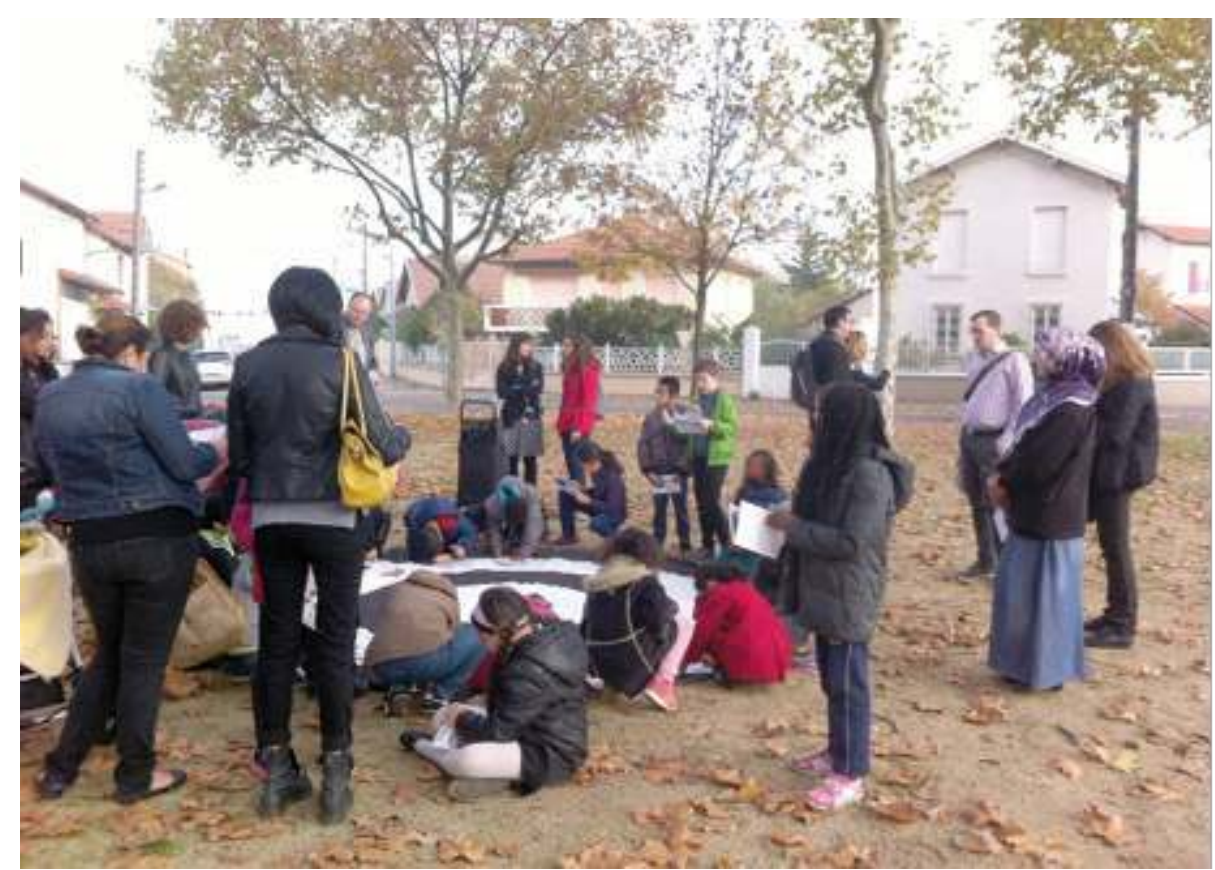

(C) G.Lesaffre

Alors que sur le papier, les ateliers en petits groupes pouvaient apparaitre comme un temps privilégié de réflexion commune, ceux-ci ont été à chaque fois détournés. À Montréal, une dynamique proche du conseil a émergé, les membres de l'institution extérieure se positionnant en pourvoyeurs d'idées irrigant les membres de l'autre institution sur la manière d'occuper l'espace urbain immédiat du CHM et du Rize, une question partagée par les deux institutions. Sans doute les attentes vis-à-vis des ateliers, mais surtout la méthodologie, n'avaient pas été suffisamment définies. L'atelier à Villeurbanne a mieux fonctionné pour un des groupes dont le questionnement était très circonscrit et se situait dans le prolongement des ateliers de la première semaine, "faire franchir la porte du Rize", mais a également dérivé vers des apports d'expérience pour alimenter les problématiques posées au Rize ou au CHM autour des plateformes Rize + et du futur blog du CHM et autour de la "valorisation des collections". Au-delà du manque de précision de la méthodologie déployée dans les ateliers, ce détournement est aussi le symptôme d'un besoin d'échanger en petits groupes sur des questions qui se posent très concrètement, qui n'a pas forcément besoin de passer par des ateliers créatifs ou d'imagination pour se réaliser.

\section{Le jeu des différences pour se situer}

Les travaux, déjà anciens de Stefan Szuman (1951) portant sur la comparaison et sa place dans la pensée analytique chez l'enfant, montrent que la comparaison est une fonction primordiale dans presque tous les process de pensée, et que comparer, c'est élargir l'horizon de sa pensée. Il n'est alors pas anodin de souligner qu'une partie importante des échanges entre les deux institutions parties prenantes, et avec les partenaires visités ou rencontrés, a consisté à comparer les échelles d'intervention, les équipements, les 
problématiques propres à chaque établissement, une explicitation nécessaire pour se situer.

La "visite des murales" de Montréal menée par l'Autre Montréal.

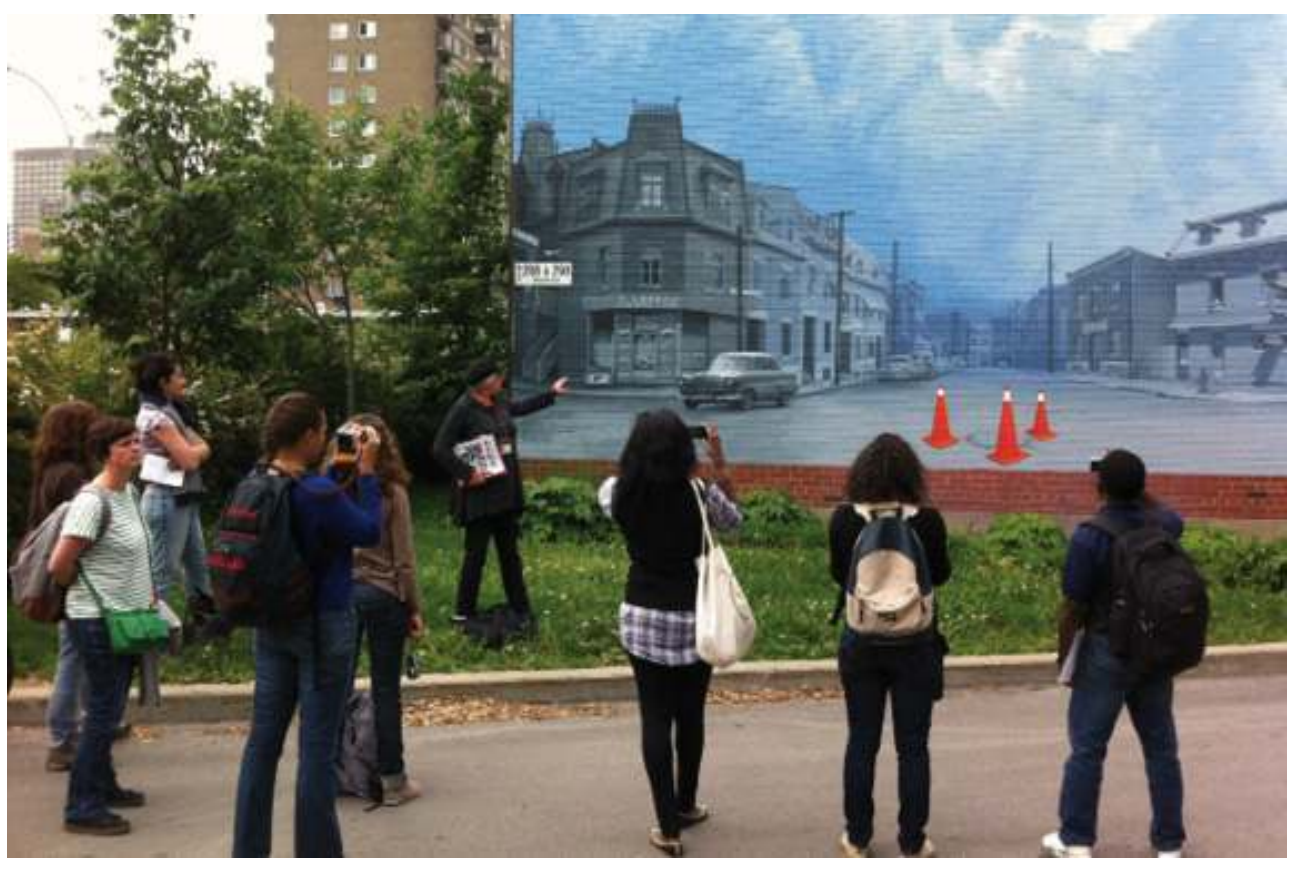

(c) G. Lesaffre par le CHM - et la ville de Villeurbanne - même si elle est intégrée à la métropole lyonnaise - ont été très fortement ressenties par les professionnels Montréalais lorsque le Rize leur a été présenté à Montréal, mais leur venue à Villeurbanne a atténué cette impression, ce qui permet de souligner l'importance de la réciprocité de l'échange pour une meilleure compréhension des enjeux des partenaires. parce qu'elles impactent l'organisation mais aussi la programmation des deux institutions. De manière générale le CHM manque de place pour ses activités autres que les expositions, ce qui changera avec le projet de déménagement. Il ne dispose pas d'auditorium et voit ses bureaux installés à l'extérieur du musée, dans un bâtiment administratif de la municipalité. Le Rize, lui, n'a pas d'espace d'exposition permanente mais dispose d'un grand auditorium qui lui permet de présenter des spectacles, et est associé aux archives de la ville et à une médiathèque municipale. Il accueille également des chercheurs.

Cette pluriactivité du Rize est posée comme une de ses problématiques fortes, et distincte de celle du CHM, qui constitue une ressource et impacte son organisation; tandis que les problématiques soulevées par le CHM concernent plutôt son environnement fortement concurrentiel, en raison de la multiplication des musées consacrés à l'histoire de Montréal ${ }^{6}$.

23 Les différences dans la constitution des équipes, en partie corrélées avec les différences d'équipements, et la répartition des missions et des tâches sont aussi interrogées. Chacun 
cherche son double au sein de l'équipe partenaire, ce qui permet de révéler les stratégies, davantage tournées vers la conservation des collections à Montréal, plus vers le développement des relations avec les publics autres que les publics scolaires ou captifs pour Villeurbanne.

Enfin, au-delà du rapprochement entre les deux partenaires, le Rize et le CHM ont également pu se situer par rapport à d'autres institutions lors des rencontres organisées, proches en raison des dynamiques de création des musées, du territoire, de la démarche, de la relation aux collections... Ces rencontres ont permis de sortir du jeu des différences fermé sur les deux partenaires et de prendre conscience d'autres rapprochements possibles. Le CHM se situe ainsi lui-même à la croisée du Rize et des musées Gadagne, et le Rize entre le CHM et l'écomusée du Fier Monde.

\section{Le jeu des différences pour définir une culture professionnelle commune}

Les échanges centrés sur les ressemblances et les différences ont surtout permis d'entrer dans le cœur du sujet en interrogeant le partage d'une culture professionnelle commune, d'abord à travers le vocabulaire employé pour désigner les objets et les pratiques liées à l'activité de l'institution, mais aussi à travers le point de vue porté sur les objets et les pratiques des deux institutions.

La comparaison des éléments de vocabulaire, au-delà de la différence entre le français du Québec et le français de France, permet ainsi de révéler que certaines expressions ne sont pas partagées par les professionnels en France ou au Québec, mais propres à l'institution, comme les "temps forts" du Rize qui qualifient une programmation thématique, ou les "cliniques de mémoire" qui constituent, au CHM un dispositif mobile de recueil de la mémoire orale.

Elle permet aussi de révéler les différences entre des expressions partagées par les secteurs professionnels en France ou au Québec. Ainsi, la "médiation culturelle" en France est entendue dans un sens plus large qu'au Québec, où la médiation désigne plutôt les projets collaboratifs menés avec des artistes. L'équivalent québécois de la "médiation" à la française serait plutôt "l'action éducative" qui désigne, pour les Français, les actions destinées au jeune public, voire au public scolaire. Quant à ce qui constitue à la fois l'objet et le public des deux institutions, il est davantage désigné comme le "citoyen" au Québec et comme l'"habitant" à Villeurbanne, traduisant, au-delà des différences de vocabulaire, des relations politico-sociales différentes entre les institutions et leur public.

Plus largement, le point de vue des institutions sur leurs objets et leurs pratiques témoigne des différences politiques et de pratiques professionnelles en France et au Québec. Ainsi, le rapport à l'immigration, ancienne ou contemporaine, a fait partie des sujets de discussion récurrents et interroge la place de l'histoire, de la mémoire et des publics immigrés dans leur relation à l'institution et, plus largement, dans la société française ou québécoise. Le CHM proclame ainsi "Vous faites partie de l'Histoire !" aux nouveaux arrivants comme à l'ensemble des habitants de la ville de Montréal, interrogeant le point de vue français. Le statut des témoignages recueillis auprès des "citoyens" ou des "habitants", pratique partagée par les deux institutions, révèle également de profondes différences de pratiques. Là où les témoignages recueillis par le CHM - qu'ils fassent partie d'une collecte dans le cadre d'une exposition temporaire, 
comme Scandale! ou dans le cadre d'un programme éducatif à destination des jeunes arrivants au Québec - sont inventoriés en tant que tels dans les collections du musée, parfois associés à l'image d'un objet lié au témoignage. Le Rize ne conservant pas de collections matérielles, la question ne s'y pose pas en ces termes, mais le statut des entretiens d'habitants collectés dans le cadre des expositions du Rize ne fait pas l'unanimité parmi les professionnels de l'institution.

Le Centre d'Histoire de Montréal anime un programme éducatif d'intégration des élèves en classe d'accueil "Vous faites partie de l'histoire !".

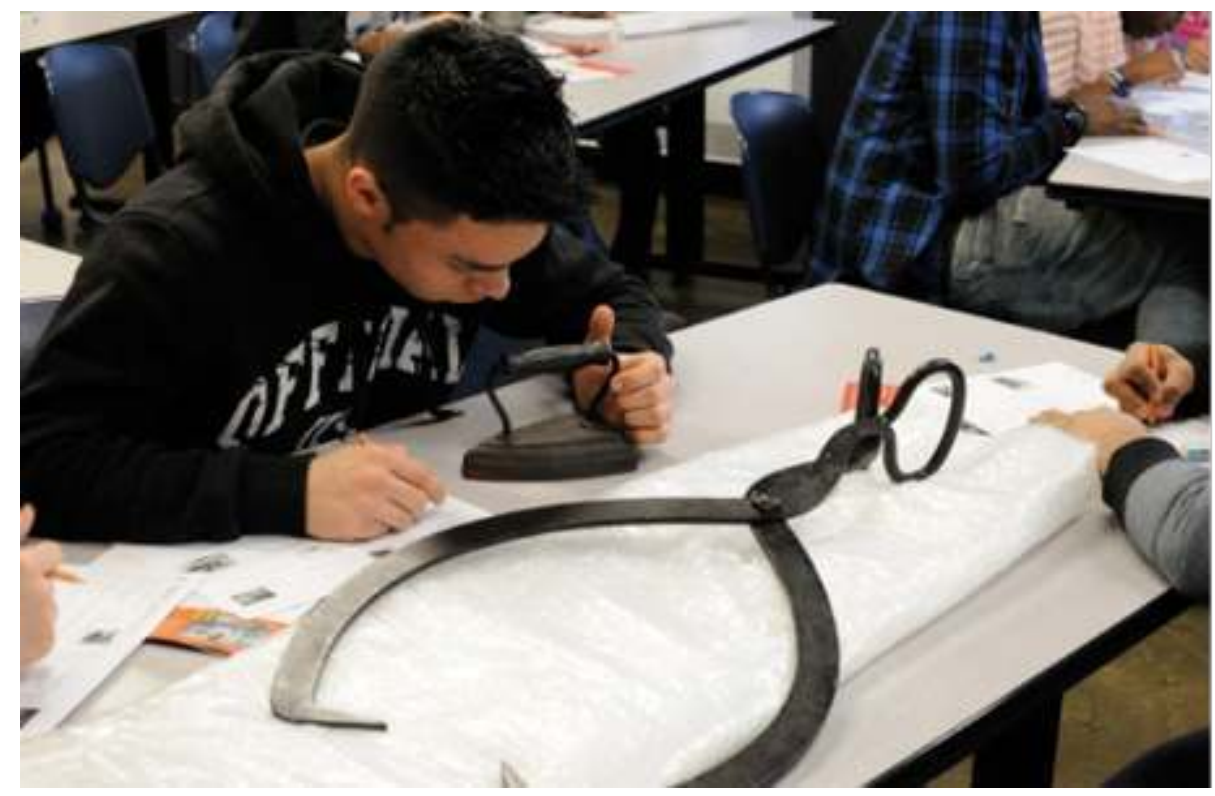

(c) Centre d'Histoire de Montréal

La compilation de ces différences est bien entendu l'occasion de pointer les ressemblances, et ainsi de conforter l'intérêt de la rencontre. La première d'entre-elles : l'année de construction des bâtiments qui abritent le CHM et le Rize, 1903, mais surtout, au-delà de l'anecdote, l'approche de l'histoire et de la mémoire tournée vers la mémoire orale des habitants et sa collecte, davantage développée et organisée au CHM, et la volonté de toucher les habitants, davantage développée au Rize.

\section{Glissement de la question de l'animation urbaine vers la valorisation de la mémoire orale}

La longueur de la liste des ressemblances et des différences laisse imaginer l'espace qui y a été consacré au sein des échanges durant les semaines montréalaise et villeurbannaise et peut faire craindre que le sujet de l'échange "L'histoire, la mémoire et le patrimoine au cœur de l'animation urbaine" n'ait été abordé. Or, non seulement la question de l'animation urbaine a été réellement posée, notamment parce qu'elle préoccupe l'équipe montréalaise, moins rompue que l'équipe du Rize à cette démarche, mais investie dans une dynamique d'animation des abords immédiats du musée, et plus largement dans le souhait d'entrer en relation avec les habitants de Montréal autrement qu'à travers les expositions; mais une autre grande question a émergé au fil des échanges, celle de la valorisation des archives orales recueillies, plus maitrisée au CHM qu'au Rize; et en 
périphérie celle de l'animation de la plateforme en ligne du Rize, Rize + et du blog du CHM.

C'est, finalement, au moment de soulever les sujets sensibles pour les uns et plus maîtrisés pour les autres, après s'être défini soi-même au regard de l'autre, que l'échange peut concerner davantage les pratiques professionnelles en tant que telles que les institutions et leur fonctionnement.

\section{Les bénéfices de la rencontre}

L'expérience vécue par les équipes du Centre d'Histoire de Montréal et du Rize de Villeurbanne montre que les vertus de l'échange effectif sont nombreuses, même si elle demande un investissement important.

D'abord, elle permet de faire un effort d'explicitation sur soi-même et donc de mieux se connaître en s'exposant aux autres, non pas dans une présentation courte, comme l'opportunité peut en être donnée dans un colloque ou une journée professionnelle, mais sur un temps long. Le nouveau directeur du Rize, très impliqué dans l'accueil de l'équipe québécoise, a indirectement pu bénéficier de cette précieuse explicitation au sein même de son institution.

Bien entendu, elle permet à deux institutions de mieux se connaître, à l'échelle des structures, mais aussi à l'échelle des réseaux de partenaires, et à l'échelle des actions menées et des démarches dans lesquelles elles s'inscrivent, comme le recueil de la mémoire orale, et surtout des individus qui mènent ces actions et ces démarches.

La rencontre permet aussi de se rassurer, notamment en se rendant compte que les autres se trouvent face aux mêmes difficultés, se posent les mêmes questions, et donc en permettant de relativiser; mais aussi en mettant en valeur ce qui fonctionne bien et les problèmes qui ne se posent pas.

Enfin, la rencontre amène bien à un échange de pratiques et constitue la source d'inspiration inscrite dans son projet même. Très concrètement, les brigades mobiles mises en place par le Rize pour interpeler les habitants dans l'espace public, inspirées de l'éducation populaire, intéressent fortement le directeur du CHM. L'existence d'un poste de "chargé de développement des publics et des projets partagés", d'agent de liaison" comme le désigne le directeur du CHM, séduit également l'équipe québécoise. "Les cliniques de mémoire", dispositif mobile de recueil de la mémoire orale élaboré par le CHM et clin d'œil au collectes de sang, inspirent l'équipe du Rize qui s'interroge déjà sur le moyen de se l'approprier: "on ne sera peut-être pas en blouse blanche! (comme les Montréalais)". Inversement, le processus de désignation du patrimoine par l'écomusée du Fier monde, et le dispositif "Vous faites partie de l'histoire!" soulignent, pour les Villeurbannais, un côté pragmatique inspirant des Québécois, tandis que l'expérimentation de médiations menées sur le parvis du musée durant l'été 2015 met l'accent sur leur capacité enviée de "faire" même si l'on n'est pas complètement prêt.

Un des résultats, sans doute moins attendu, de la rencontre est qu'elle amène à relativiser le caractère innovant de certaines des actions menées. Là où les équipes pensent parfois être isolées et à la pointe dans leurs pratiques, la rencontre avec d'autres permet de mettre en perspective les actions menées et ainsi de rompre l'impression d'isolement et de relativiser leur caractère d'exception, tout en confortant les choix réalisés et la pertinence des actions menées. 


\section{Ce n'est qu'un début}

\begin{abstract}
a progressivement glissé des directeurs et des institutions aux équipes. Les professionnels impliqués sont maintenant susceptibles d'entretenir des échanges individuels en fonction des tâches et des préoccupations de chacun, de dépasser le collectif pour entretenir des relations de pair à pair, l'échange ayant permis d'instaurer la relation de confiance nécessaire à la poursuite de l'échange, mais aussi de mieux définir ce que chacun pouvait attendre de l'autre. Le risque d'en rester là est néanmoins soulevé. Il faut se donner des objectifs pour entretenir la relation. Plusieurs sont évoqués comme une veille réciproque, mais aussi de nouvelles pistes de travail qui ont émergé pendant les échanges, autour du développement de l'usage de la plateforme Rize + et du blog du CHM, et autour de la mise en exposition et du catalogage des témoignages.
\end{abstract}

sue de ces deux semaines d'échanges entrecoupées d'une pause estivale, la rencontre

\section{De la conversation au jumelage : genèse d'un projet}

L'idée d'un échange professionnel entre le Centre d'histoire de Montréal et le Rize est d'abord le fruit d'une rencontre humaine. Peu de temps après l'ouverture du Rize au public, j'ai eu l'occasion de m'investir dans un groupe de réflexion piloté par le ministère de la Culture autour des questions de patrimoine et d'interculturalité. Le sujet, plus que jamais d'actualité, sur la lancée de l'année européenne du dialogue interculturel (2008), réunissait chercheurs, services d'archives, bibliothèques et musées. En 2011, ce collectif s'étend aux associations et se structure sous la forme d'un groupement d'intérêt scientifique intitulé "Institutions patrimoniales et pratiques interculturelles" (Ipapic). J'en deviens alors le président, tandis que se mettent en place les instances qui vont l'animer, avec notamment un comité scientifique auquel vont participer des chercheurs, des responsables d'établissements et des représentants d'associations. Jean-François Leclerc, directeur du Centre d'Histoire de Montréal, accepte d'en être membre. Et c'est ainsi que nous aurons l'occasion de nous croiser à plusieurs reprises, dans le cadre des nombreux séminaires, rencontres et ateliers qui sont organisés jusqu’à la fin du Gis Ipapic en septembre 2015.

Nous avons débuté une conversation fructueuse, reprenant en pointillé d'une année à l'autre nos discussions sur les sujets qui nous étaient communs et nos préoccupations réciproques. Constatant ainsi la proximité de nos questionnements et de nos pratiques, nous avons fait germer ensemble l'idée de construire une sorte de jumelage entre nos deux institutions. Nous avons donc saisi l'opportunité de "l'Accord France-Canada pour la coopération et les échanges dans le domaine des musées", qui a fourni le cadre du projet d'échange. Le projet avait eu le temps de murir et a pu trouver son éclosion en 2015.

Xavier de la Selle,

Directeur des musées Gadagne depuis mai 2015 


\section{L'échange avec le Rize : un partage de valeurs communes}

Les professionnels français et québécois se fréquentent et échangent des idées depuis longtemps. Leur langue commune et les questions partagées facilitent les contacts. Mais nous savons d'expérience qu'il est souvent difficile de transformer les liens cordiaux établis lors de colloques, en relations soutenues et en projets communs qui transformeront nos cultures institutionnelles et nos pratiques. La distance, le temps, l'hyperactivité muséale, les budgets et tant d'autres contraintes sont souvent en cause, plus que la bonne volonté des personnes concernées. Pourtant, le monde muséal ne manque pas de défis qui demandent des solutions innovates, lesquelles auraient plus de chance d'émerger d'une réflexion lentement mûrie et partagée. En effet, dans nos milieux professionnels tissés serrés et au sein de nos cultures nationales, un certain consensus conforte souvent nos certitudes et masque nos lacunes, surtout quand un certain succès public ou professionnel est au rendez-vous. Il est donc essentiel de nous confronter périodiquement à ce qui ne nous est pas familier, à d'autres manières de voir et d'agir.

L'exposition permanente Traces. Lieux. Mémoires consacrée à l'histoire de Montréal, des glaciers à aujourd'hui.

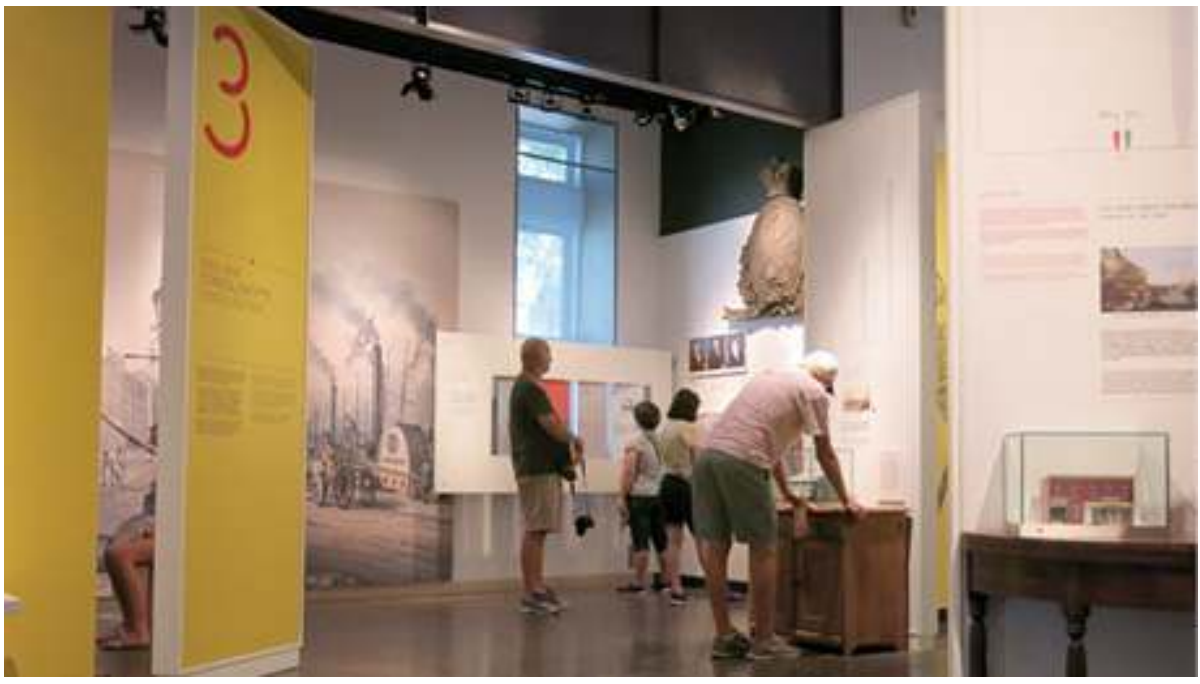

(c) Centre d'Histoire de Montréal

L'occasion se présenta dans le cadre du Gis Ipapic, suite à l'invitation d'Hélène Hatzfeld, du ministère de la Culture, qui coordonnait ce groupe. J’y rencontrai Xavier de La Selle, alors directeur du Rize, qui me fit découvrir sa jeune institution de Villeurbanne (et son équipe). J'ai rapidement senti que le Rize partageait avec le Centre d'Histoire de Montréal (CHM) des valeurs, une philosophie d'action, un statut municipal et une mission résolument urbaine. Le projet d'entreprendre des échanges plus soutenus fut donc maintes fois envisagé, tout en sachant que des siècles d'histoire, une culture muséale, des échelles urbaines très différentes et un océan séparaient Montréal et Villeurbanne. Pour le Centre d'Histoire, il s'agissait de s'inspirer des activités imaginées par le Rize pour entrer en relation avec la population, en particulier dans la rue ou sur les places publiques. Le CHM commençait en effet à préparer son déménagement du paisible Vieux- 
Montréal vers un lieu très animé du centre-ville. Il nous semblait important d'explorer diverses voies pour devenir d'une certaine manière, un "musée-rue" ou un "musée de la rue", qui prolongerait la voie publique et qui pouvait prendre place dans le quotidien des passants du centre-ville. Notre porte-folio était certes riche en actions entreprises avec divers groupes de la société, mais dans le domaine de l'animation sur rue, il fallait, et il faut encore, faire nos classes, car le contexte immédiat du CHM dans le quartier historique de même que ses ressources n'avaient pas permis de développer cette expertise.

\section{Le Centre d'Histoire de Montréal}

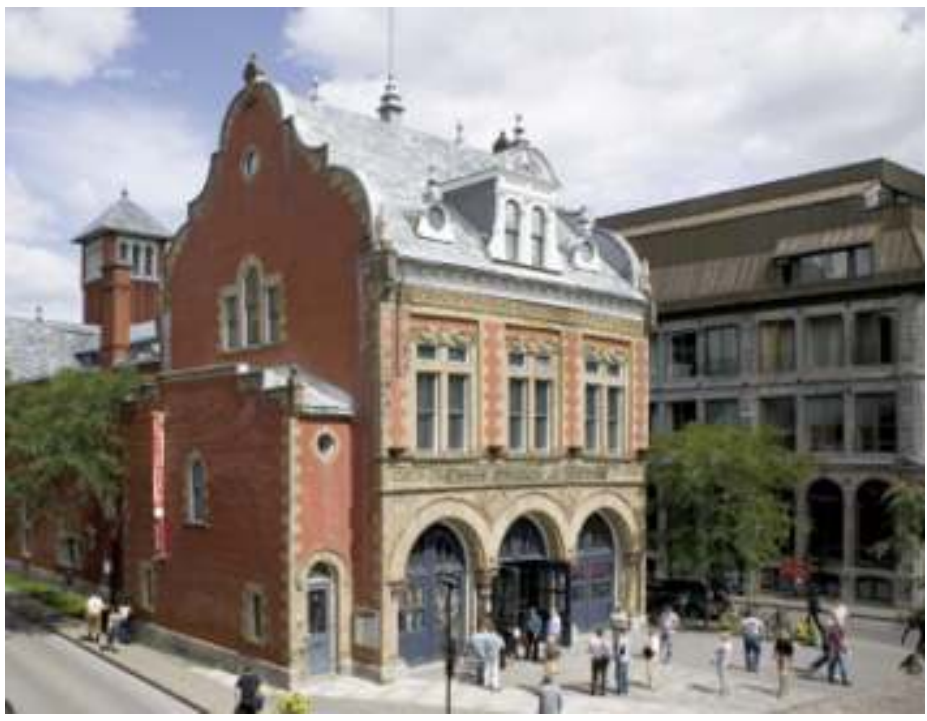

(c) Normand Rajotte/Centre d'Histoire de Montréal

Le projet d'échange fut donc conçu dans le cadre du programme Canada-France pour nous permettre de partager nos expériences en projets hors les murs. En 2015, les représentants du Rize et du CHM firent connaissance à Montréal puis à Villeurbanne par des visites, des séances de travail en équipe, des rencontres avec des partenaires et la mise en commun devant public. Du côté du CHM, une muséologue, la responsable des programmes éducatifs et un guide-animateur se rendirent en France à l'automne 2015. Nos premières impressions quant aux similitudes furent-elles confirmées? Oui, tout autant que celles sur nos différences, comme le rapporte Gaëlle Lesaffre dans le présent article. Saluons au passage l'heureuse initiative du Rize d'avoir demandé à une observatrice indépendante d'accompagner nos échanges, un atout pour la qualité et l'impartialité du bilan. Chose certaine, les uns ont retrouvé chez les autres la même volonté d'établir avec les citoyens/habitants de nos agglomérations une relation plus directe, plus ouverte, faite d'écoute et de collaboration. Xavier étant devenu directeur des musées Gadagne, il décida d'engager dans le projet d'échange cette institution lyonnaise dont certains traits, différents du Rize, le rapprochent du CHM. Mentionnons aussi que Montréal et Lyon sont des villes jumelées depuis 1989. J'ai par la suite rencontré Vincent Veschambre, nouveau directeur du Rize, et retrouvé la même cordialité des premiers contacts avec Xavier. Ce sont donc trois institutions qui, en 2017 et 2018, se lanceront un défi: expérimenter sur nos propres terrains, une activité bien rodée de l'un des partenaires, en l'adaptant à son propre contexte, et en analysant conjointement le processus et les résultats. La créativité des musées contemporains mérite en effet que 
leurs innovations circulent et inspirent des collègues de partout dans le monde. Espérons que cette expérience permettra de savoir comment le faire et bien le faire, au profit de toute la communauté muséale.

\section{Le croisement avec le Centre d'Histoire de Montréal : quel impact pour le Rize?}

Le Rize est implanté au cœur de la zone urbaine villeurbannaise.

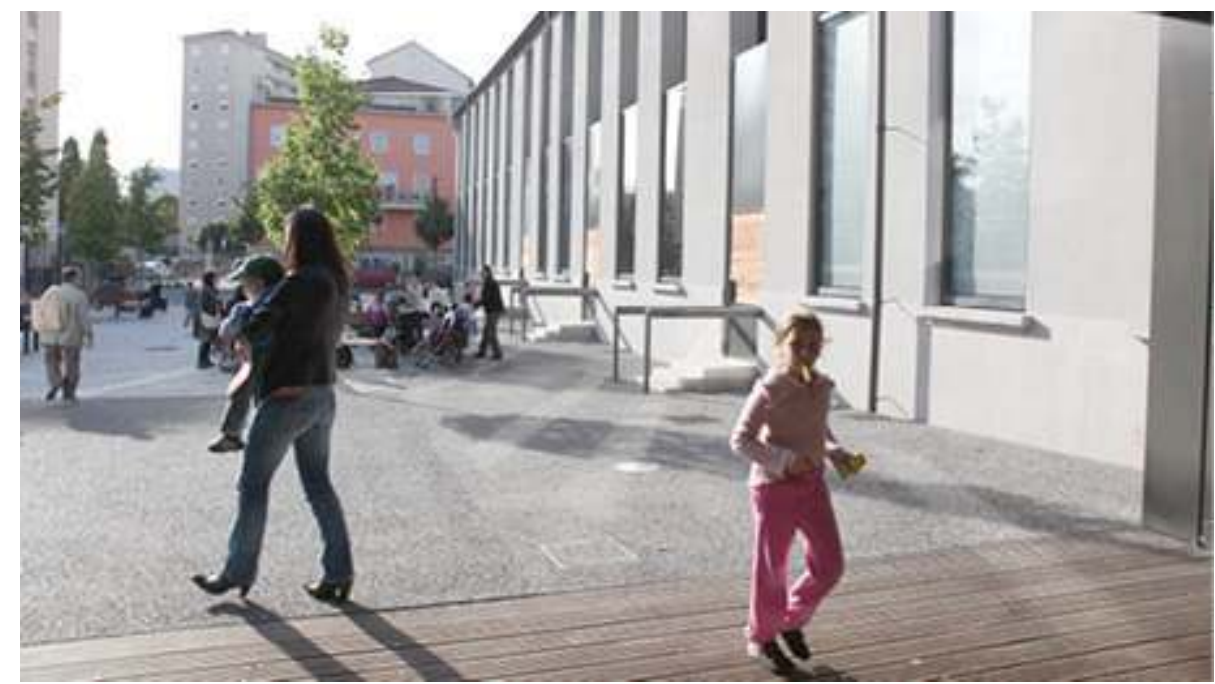

(c) Le Rize/Ville de Villeurbanne

En tant que directeur nouvellement installé, l'accueil d'une partie de l'équipe du CHM en novembre 2015 a été l'occasion d'entrer de plain-pied dans un échange voulu par mon prédécesseur, Xavier de la Selle, échange dont j'ai tout de suite perçu le caractère structurant pour le Rize. À travers cette rencontre qui conjugue altérité et proximité, il y a de quoi nourrir la réflexion pour les années à venir, alors même que mon équipe est engagée dans l'élaboration de son projet de service pour les quatre années qui viennent.

\section{Des décalages significatifs mais nourrissants}

L'échange a confirmé un certain nombre de décalages prévisibles entre les équipements.

Le géographe que je suis (et qui a eu l'occasion de découvrir Montréal et le CHM) est tout d'abord sensible aux différences d'échelles entre les territoires de référence de nos institutions. Entre la deuxième commune de l'agglomération lyonnaise et la métropole québécoise, l'écart peut paraître considérable, voire rédhibitoire. Réinscrire Villeurbanne dans son cadre métropolitain réduit considérablement le différentiel : 1584738 habitants pour l'agglomération lyonnaise en 2012, 1988243 habitants pour celle de Montréal en 2014. Même s'il ne faut pas perdre de vue que le Rize travaille sur et avec le territoire communal, les questions sociales et culturelles abordées sont bien celles d'une métropole de taille européenne, qui se situe certes un cran en dessous de Montréal (métropole de 
taille mondiale) mais avec laquelle la comparaison peut avoir du sens: je pense en particulier à la question migratoire qui est structurante dans les deux cas. Par ailleurs, cette question d'échelle nous a fait prendre conscience de l'intérêt d'élargir pour la suite la partie française à une institution lyonnaise : le partenaire en est tout trouvé, étant donné que l'actuel directeur des musées Gadagne se trouve être l'ancien (et premier) directeur du Rize, à l'origine de cet échange.

Des différences également dans le rapport aux expositions et aux collections peuvent également faire apparaître nos deux équipements comme différents. Alors que le CHM présente une exposition permanente sur la ville, le Rize fonctionne uniquement avec des expositions temporaires. Pour les expositions temporaires, les temporalités sont différentes : de l'ordre de plusieurs mois au Rize et de plusieurs années au CHM. Le CHM gère ainsi une collection, comme un musée "classique", alors que parler de collections au Rize renvoie spécifiquement aux archives municipales, voire au fonds local de la médiathèque qui est en cours de structuration et de développement : ce qui induit pour le Rize une moindre familiarité à l'objet, mais une plus grande proximité avec les archives. Mais plus que des freins à la rencontre, ces différences en matière de "collections" permettent à chacun de faire un pas de côté pour s'interroger sur ses manques potentiels et d'aller chercher du côté du partenaire, des ressources et compétences qui font défaut, que ce soit pour mener des collectes, conserver des objets ou pour alimenter un site ou un blog.

\section{Des points communs qui permettent d'entretenir une affinité}

53 Par-delà ces différences d'environnement, de contexte institutionnel, de missions, les préoccupations paraissent suffisamment proches pour alimenter cet échange. Tout d'abord dans la manière d'appréhender le territoire de référence comme matière première : il s'agit bien de donner à voir, à comprendre un territoire, dans sa diversité. Même si le CHM a des collections, le travail de gestion de ces collections ne représente qu'une toute petite partie du travail, qui est prioritairement tourné vers l'extérieur. Quant au Rize, musée sans collection, il n'a d'autre choix que de se penser comme musée de territoire, dont le territoire, ses paysages, ses constructions, mais aussi ses habitants et ses pratiques sociales constituent de fait "la collection", une collection dynamique, à réinventer en permanence. 
L'opération "Graines de mémoire" développée par Le Rize au printemps 2016 est une adaptation du projet "Les Cliniques de mémoire", collectes de mémoire hors les murs organisées par le Centre d'Histoire de Montréal.

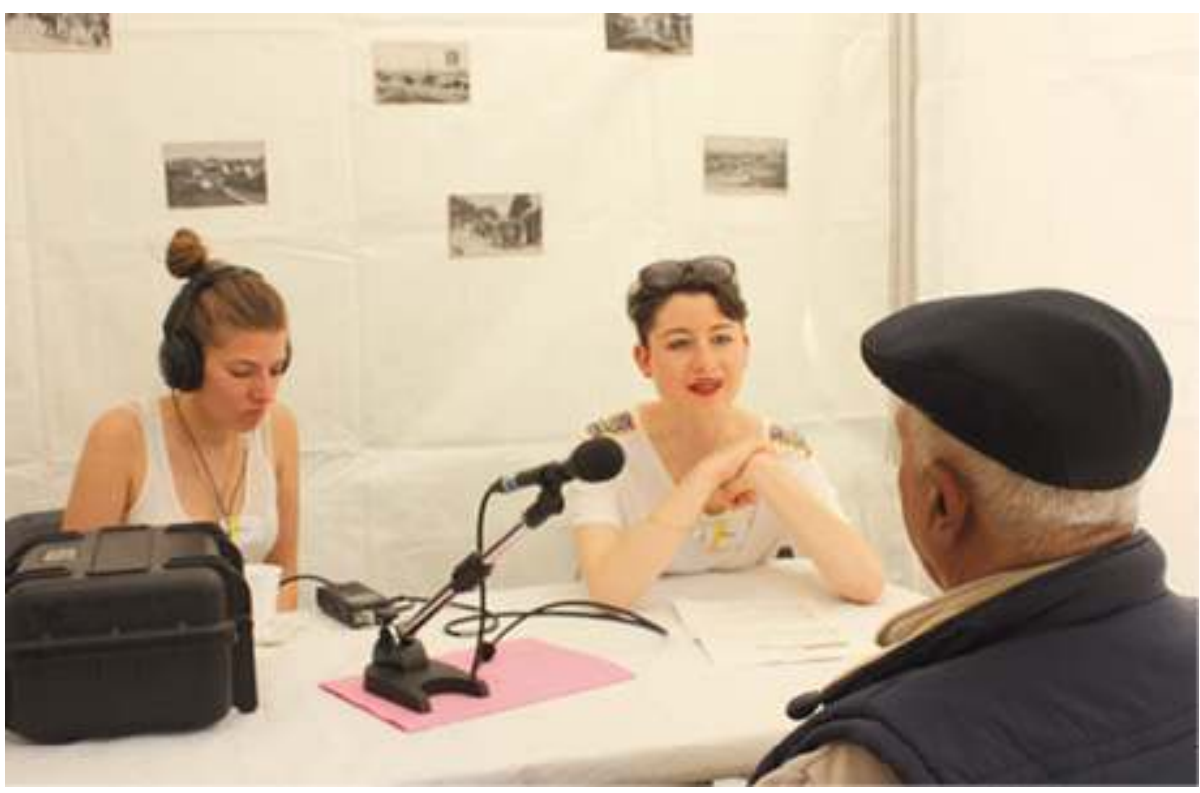

(C) Le Rize/Ville de Villeurbanne

54 J'y faisais allusion plus haut, la référence aux apports migratoires dans la constitution d'une société urbaine multiculturelle constitue également un ciment important entre nos deux équipes. Avec certes des traditions politiques et culturelles contrastées d'un contexte à l'autre mais c'est aussi cela qui permet de porter un regard renouvelé sur sa propre société.

Enfin, dans les deux cas, la priorité est mise sur le principe de "travailler avec plutôt que pour", pour reprendre une formule mise en exergue par Xavier de la Selle. Il s'agit d'inventer des dispositifs qui mettent les habitants en situation d'acteurs, pour raconter avec nous une histoire collective.

La fête du sport

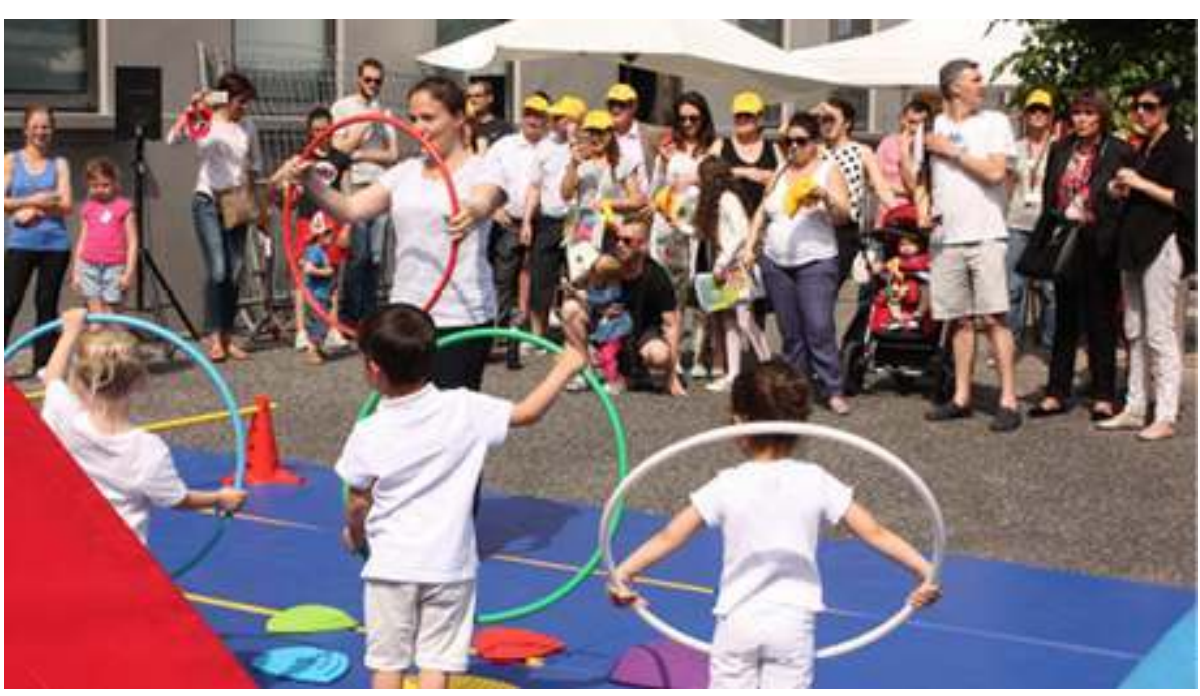

(c) Le Rize/Ville de Villeurbanne 
Au final, ce qui m'apparaît le plus stimulant dans ce que j'ai perçu de cet échange, c'est ce qui relève de la prise de conscience des points forts (et inversement de ses propres limites ou faiblesses) chez le partenaire, ce qui donne matière à s'appuyer sur l'expérience que ce partenaire a développée pour progresser soi-même, alors même que les objectifs fondamentaux sont les mêmes. C'est ainsi qu'il ressort de ce premier échange que la collecte de la mémoire orale des habitants est davantage développée et organisée au CHM, tandis que les méthodes pour aller à la rencontre des habitants semblent plus éprouvées au Rize : ce constat est de nature à alimenter des échanges pérennes.

\section{Une suite, dans un esprit de jumelage?}

Ayant fait le constat du caractère à la fois inachevé et stimulant de cet échange, j'ai souhaité que nous répondions à l'appel à projet France-Canada du ministère de la Culture, qui avait permis il y a deux ans de lancer ce processus de rencontre.

Afin de se donner les moyens et le cadre nécessaires pour suivre les effets de ce premier d'échange, pour observer la manière dont les équipes s'emparent des méthodes, des pratiques qu'elles ont jugées éprouvées et pertinentes chez le partenaire, leur permettant de combler des faiblesses identifiées ou d'innover dans un domaine jugé prioritaire. En d'autres termes, il apparaît judicieux d'analyser les formes de transposition, d'interprétation, d'appropriation de ces actions qui ont nourri la réflexion dans un premier temps et qui vont être assimilées, intégrées dans les projets et mises en pratiques dans les mois et les années qui viennent. Chaque institution a ainsi identifié une ou deux actions qu'elle prévoit de mettre en œuvre à court terme, action nourrie par l'échange précédent et d'ailleurs repérée dans le cadre du travail de Gaëlle Lesaffre. Pour les musées Gadagne, que nous avons intégrés comme troisième partenaire dans cette réponse, la connaissance précise dont dispose le directeur lui permet d'identifier de la même manière des actions dont il prévoit de s'inspirer dans les mois et années à venir pour sa propre institution.

Un tel suivi des effets de ce premier échange présente un intérêt à la fois scientifique, puisqu'il permet d'approfondir l'analyse des différences de contextes (politiques, professionnels, sociaux...) et très appliqué, puisqu'il va structurer la mise en œuvre d'actions jugées prioritaires par les institutions partenaires.

\section{Le croisement et la transposition des pratiques}

Dans la logique du diagnostic réalisé lors de la première phase d'échange, le Rize souhaite s'inspirer des "cliniques de mémoire", dispositif mobile de recueil de la mémoire orale élaboré par le CHM et clin d'œil aux collectes de sang, tout en cherchant à sortir de la référence médicale qui semble moins adaptée au contexte français. C'est du côté de la métaphore végétale qu'il s'oriente, ayant programmé une première collecte de "graines de mémoire" au printemps. Du côté du CHM, ce sont les "brigades mobiles" utilisées pour provoquer la prise de parole dans l'espace public et nourrir des expositions, qui font l'objet d'un intérêt manifeste. L'élargissement du partenariat à Gadagne enrichit encore le dispositif, son directeur n'ayant eu aucune difficulté à identifier les points forts du CHM et du Rize dont il souhaite s'inspirer. 
61 Dans le même temps, et dans une logique comparative, le CHM, le Rize et les musées Gadagne vont tester une même expérience de travail avec des enfants issus de l'immigration. Il s'agit d'une action visant à questionner la notion même de patrimoine auprès de celles et ceux qui en sont les futurs dépositaires. C'est autour des objets hérités des générations précédentes, et qu'ils considèrent comme faisant partie de leur patrimoine, que seront sollicités ces enfants. CHM et Rize ont l'habitude de questionner les patrimoines de l'immigration, ce qui intéresse particulièrement les musées Gadagne, Rize et Gadagne faisant tous deux partie de l'association Traces, qui met en réseau à l'échelle régionale les associations et institutions qui travaillent sur histoire et mémoires de migrants. Le CHM et Gadagne apporteront leur pratique des collectes et mises en récits d'objets, pendant que le Rize mobilisera son expérience des ateliers mémoriels et patrimoniaux avec les plus jeunes.

Nous sommes désormais dans l'attente d'une réponse suite au dépôt de ce projet. Nous savons dans le même temps que même si ce financement ne nous est pas accordé pour fonctionner, les liens entre les trois institutions sont suffisamment forts pour qu'ils se traduisent à terme dans d'autres dispositifs de financement et de rapprochement. Le tout étant de veiller à poser ce cadre, nécessaire pour nous contraindre à dégager des moyens et du temps, afin de nous extraire des urgences du quotidien et de nous permettre de prolonger et faire vivre des échanges particulièrement stimulants.

Vincent Veschambre,

Directeur du Rize, Ville de Villeurbanne

\section{BIBLIOGRAPHIE}

Hatzfeld, H. (dir.). Regards décalés sur des patrimoines silencieux. Paris : Les ateliers Henri Dougier, 2015, 153 p.

Grange, S. et Hatzfeld, H. Recherche et musées, une rencontre improbable ou l'interculturel comme un viatique, in Le Marec J., Maczek, E. et Lochot, S. Musées et recherche: cultiver les alliances. Dijon : OCIM, 2012, pp. 125-141.

Testart, A. Échange marchand, échange non marchand, Revue française de sociologie, vol. $42, \mathrm{n}^{\circ} 4$. 2001, pp. 719-748.

Szuman, S. Comparaison, abstraction et pensée analytique chez l'enfant, Enfance, vol. $4, \mathrm{n}^{\circ} 3$, 1951, pp. 189-216.

\section{NOTES}

1. Voir http://ipapic.eu/.

2. L'échange a pris appui sur la contribution financière apportée dans le cadre de l'Accord France-Canada pour la coopération et les échanges dans le domaine des musées. Voir : www.icom-musees.fr/uploads/media//Accord_France_Canada/CANADA_OK_25_02002-1-2.pdf.) 
3. Le groupement d'intérêt scientifique "Pratiques interculturelles dans les institutions patrimoniales" (Ipapic) se propose "de mieux connaître les défis que les dynamiques interculturelles posent aujourd'hui aux processus de patrimonialisation, de mieux les comprendre pour dessiner des pistes d'action tant en matière de projet d'établissement que de politiques culturelles. Il vise à ouvrir de nouveaux champs de recherche en sciences humaines et sociales, à expérimenter des modes collaboratifs de recherche, à favoriser la mise en cuvre et la diffusion de pratiques nouvelles dans les institutions patrimoniales. Lieu de décloisonnement dans les modalités de production des connaissances, notamment entre chercheurs et autres producteurs de savoir, le Gis Ipapic se veut un laboratoire permanent à partir des interrogations du groupe de travail." Voir www.ipapic.eu/presentation/objectifs/

4. Le CHM ne compte pas de responsable du développement des publics et des projets partagés, mais celle du Rize devait faire partie du voyage dès le départ.

5. Écomusée du Fier monde, musée des Maîtres et Artisans du Québec, L'Autre Montréal, musées Gadagne, le CCO, les gratte-ciels de Villeurbanne.

6. Musée McCord, musée Stewart, musée Pointe-à-Callière, écomusée du Fier monde, musée du Château Ramezay, musée Marguerite-Bourgeoys, musée de Lachine, cité Historia-musée du Saultau-Recollet.

\section{RÉSUMÉS}

Au-delà des différences de contexte, d'échelle territoriale et de vocabulaire, la rencontre entre les équipes du Centre d'Histoire de Montréal (CHM) et du Rize de Villeurbanne a permis de mettre en perspective les actions menées et les difficultés rencontrées au quotidien dans les pratiques mais elle a surtout constitué une source d'inspiration et de motivation en donnant une occasion de nouer des liens entre professionnels pour poursuivre l'expérience.

\section{INDEX}

Mots-clés : échange de pratiques

\section{AUTEUR}

\section{GAËLLE LESAFFRE}

docteure en Sciences de l'Information et de la Communication, responsable de Public, Culture et Numérique

lesaffregaelle@gmail.com 